\title{
Algorithm for Resonator Parameter Extraction From Symmetrical and Asymmetrical Transmission Responses
}

\author{
Patrick Krkotić, Student Member, IEEE, Queralt Gallardo, Nikki Tagdulang, Montse Pont, and Juan \\ M. O'Callaghan, Senior Member, IEEE
}

\begin{abstract}
We describe an algorithm capable of extracting the unloaded quality factor and the resonant frequency of microwave resonators from vector Sparameters. Both symmetrical (Lorentzian) and asymmetrical (Fano) transmission responses are supported. The algorithm performs an adaptive outlier removal to discard measurement points affected by noise or distortion. It removes the effects caused by imperfections in the device (such as modes with close resonance frequencies or stray coupling between the resonator ports) or the experimental setup (such as lack of isolation or dispersion in the test-set and cables). We present an extensive assessment of the algorithm performance based on a numerical perturbation analysis and on the evaluation of S-parameter fitting results obtained from network analyzer measurements and resonator equivalent circuits. Our results suggest that uncertainty is mainly caused by factors that distort the frequency dependence of the S-parameters, such as cabling and coupling networks and is highly dependent on the device measured. Our perturbation analysis shows improved results with respect to those of previous publications. Our source code is written in Python using open source packages and is publicly available under a freeware license.
\end{abstract}

Index Terms-Microwave resonators, quality factor, resonant frequency.

\section{INTRODUCTION}

$\mathrm{A}$ CCURATE determination of quality factor and resonant frequency in microwave resonators is key in many applications such as measurement of material properties, design of microwave filters and fundamental physics experiments.

Resonators are known to be the most sensitive devices to measure permittivity, permeability and surface impedance of low-loss materials [1]-[4]. While there are many techniques and resonator designs, all of them rely on measuring the resonator quality factor $\left(Q_{0}\right)$ and resonant frequency $\left(f_{0}\right)$ to obtain the

Manuscript submitted March $22^{\text {nd }}, 2021$. This work was supported by CERN under Grants FCC-GOV-CC-0210 (KE4945/ATS) and FCC-GOV-CC-0209 (KE4946/ATS). UPC funding was also provided through the Unit of Excellence María de Maeztu MDM2016-0600. NT acknowledges MSCA-COFUND-2016754397 for the $\mathrm{PhD}$ grant.

P. Krkotić, N. Tagdulang and M. Pont are with ALBA Synchrotron Light Source. Carrer de la Llum 2-26 08290 Cerdanyola del Vallès, Barcelona, Spain. P.K and N.T are PhD candidates at CommSensLab, Department of Signal Theory and Communications, Universitat Politècnica de Catalunya (UPC), c/ complex permittivity, permeability or surface impedance.

Resonators are also the main building blocks of many communication devices at microwave frequencies, such as filters, diplexers and multiplexers. These are typically bandpass devices whose center frequency, passband flatness and frequency selectivity depend strongly on their resonator's quality factors and resonant frequencies [5].

Many fundamental physics applications are also based on resonators. In many cases, large quality factors are needed to achieve strong radio-frequency (RF) fields [6]. In other applications - such as axion and WISP dark matter searches [7] - resonators are used for detection, and high quality factors are required to enhance weak RF fields produced by sub-atomic particles.

Since the use of microwave resonators extends beyond the microwave engineering community, there is a need for nonexperts in the field to extract quality factor and resonant frequency from microwave measurements typically made with a vector network analyzer (VNA). We have developed an opensource, web-executable [8] Python [9] code to fulfill this need and promote the use of microwave resonators outside the engineering community. The web-based application, capable of remote execution of the code, will also prevent the frequent and unnecessary duplication of codes written by an occasional need for this functionality, but made without taking into account the vast previous work in this topic.

Our procedure combines several existing techniques based on the transmission method [10]-[15] in a single algorithm, to which we have added the following features:

1) Outlier removal, which makes the fitted results largely independent of distortion in the frequency response caused by imperfections in the measurement device and setup.

2) Ability to fit Lorentzian (symmetrical) and Fano (asymmetrical) responses.

3) Compensation of the effects produced by uneven distribution of measurement points along the resonance

Jordi Girona 1, 08034 Barcelona Spain. (emails: patrickkrkotic@,outlook.de, ntagdulang@cells.es, pont@,cells.es).

Q. Gallardo was with ALBA Synchrotron Light Source and is MS. candidate at, Department of Signal Theory and Communications, Universitat Politècnica de Catalunya (UPC), c/ Jordi Girona 1, 08034 Barcelona Spain.

J. M. O'Callaghan is with CommSensLab, Department of Signal Theory and Communications, Universitat Politècnica de Catalunya (UPC), c/ Jordi Girona 1, 08034 Barcelona Spain.(email:joan.ocallaghan@upc.edu). 


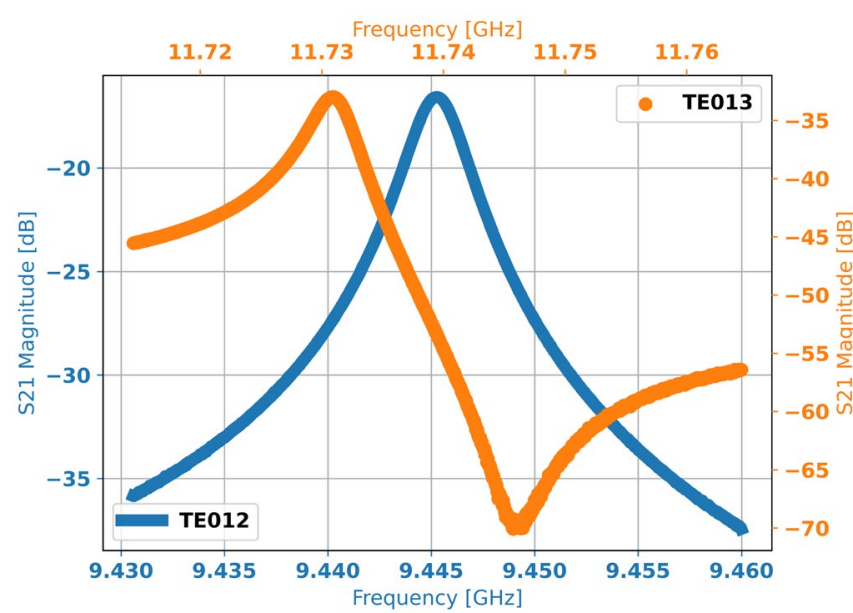

Fig. 1. Symmetrical and asymmetrical transmission response in dielectric resonator modes $\mathrm{TE}_{012}$ and $\mathrm{TE}_{013}$.

circles in the complex plane resulting from the VNA's linear frequency sweep [12], [14].

\section{FitTing PROCEDURE}

\section{A. Initial Detection of Resonance - Frequency Sweep Requirements}

The algorithm is designed to extract loaded quality factor $\left(Q_{L}\right)$ and resonant frequency $\left(f_{0}\right)$ from S-parameter data containing a maximum in the transmission response $\left|S_{21}(f)\right|$ which may be adjacent to a minimum (Fig. 1). Symmetrical resonance responses with a single maximum follow a Lorentzian curve, whereas asymmetrical ones, including those with adjacent minima, follow a Fano profile. Identifying resonances in a VNA involves locating local maxima and minima in $\left|S_{21}(f)\right|$.

To analyze symmetrical responses, the VNA frequency sweep should be centered at the position of the maximum of $\left|S_{21}(f)\right|$, include at least 20 points within the $3 \mathrm{~dB}$ bandwidth and cover a span 10 times the $3 \mathrm{~dB}$ bandwidth with at least 201 points. These settings will produce a $S_{21}(f)$ trace like the one in Fig. 2 in the VNA polar plot. In a Fano response, the trace should be identical, but shifted with respect to the origin of the complex plane [14]. Adjusting the VNA sweep parameters according to the polar plot for Fano responses requires setting the center frequency at the point of the trace with minimum density of points, placing at least 20 points covering a $180^{\circ}$ arc about the center frequency and setting a sweep with a frequency span ten times the bandwidth required to cover the $180^{\circ}$ arc above. The number of points in the frequency sweep should be at least 201.

While other settings may also prove adequate, our uncertainty analysis has been performed with the settings described above.

Note that these requirements set the maximum $Q_{L}$ given the instrument's specifications. The minimum frequency step $\Delta f$ attainable by the VNA should be smaller than one twentieth of the $3 \mathrm{~dB}$ bandwidth. This condition can be rewritten as:

$$
\frac{\Delta f}{f}<\frac{1}{20 \cdot Q_{L}} .
$$

A conservative estimate for maximum $Q_{L}$ would be to use the VNA absolute frequency accuracy in the left hand side of (1). A benchtop instrument with standard options has accuracies on the order of $10^{-6}$ due to temperature stability and aging per year, corresponding to maximum $Q_{L}$ on the order of $10^{7}$. These values can be improved by using high precision frequency options available in most VNAs, or using external frequency references.

\section{B. Linear Phase Shift Removal}

The algorithm starts by performing a phase unwrapping procedure to remove discontinuities in the S-parameter's phases. Next, their linear phase dependence [13], [14] is removed by performing a linear regression on two frequency segments, each one covering $10 \%$ of the frequency span indicated in the paragraphs above, located at the upper and lower extremes of the span.

\section{Coarse Cross-Talk Removal}

Asymmetries in the transmission response are often due to stray coupling between resonator ports. This results in an offset of the position of the $S_{21}$ trace with respect to the origin of the complex plane. Recovering the asymmetry of the transmission response can be done by re-positioning the trace in the complex plane to its theoretical position [14]. A simplified, approximate method consists in shifting the trace leaving the origin at the midpoint between its two ends, i.e.:

$$
S_{i}^{\prime}=S_{i}-\frac{S_{1}+S_{n}}{2},
$$

where $S_{i}=S_{21}\left(f_{i}\right)$ are the values of $S_{21}$ measured at discrete frequencies $f_{i}(i=1 \ldots n)$ and $S_{i}^{\prime}$ are their corresponding repositioned values. As discussed below, the small residual crosstalk effects left in $S_{i}^{\prime}$ do not degrade the fit used to obtain $Q_{L}$ and $f_{0}$.

\section{Transmission Response - Fitting Basics}

To fit the data, the algorithm makes an initial coarse estimate of $f_{0}$ by assuming it is the frequency $f_{i}$ for which $\left|S_{i}^{\prime}\right|$ is maximum. Then, the set $S_{i}^{\prime}$ is fitted using the equation below:

$$
S_{i}^{\prime}=\frac{K}{1+2 j Q_{L}\left(\delta_{i}-\delta_{e}\right)}+G
$$

where $K$ is a complex fitting parameter (the value of $S_{21}$ at resonance in absence of cross-talk), $G$ is a complex fitting parameter that takes into account residual cross-talk, $Q_{L}$ is a real fitting parameter (the resonator's loaded $Q$ ), and $\delta_{i}$ is calculated from $f_{0}, f_{i}$ :

$$
\delta_{i}=\frac{1}{2}\left(\frac{f_{i}}{f_{0}}-\frac{f_{0}}{f_{i}}\right) .
$$

Note that substituting $f_{0}$ by $f_{0}+\Delta f$ in (4) with $\Delta f \ll f_{0}$ decreases $\delta_{i}$ by a term $\delta_{e}=\Delta f / f_{0}$, thus $\delta_{e}$ in (3) is a fitting parameter that can be related to tolerances in the estimated resonant frequency. 


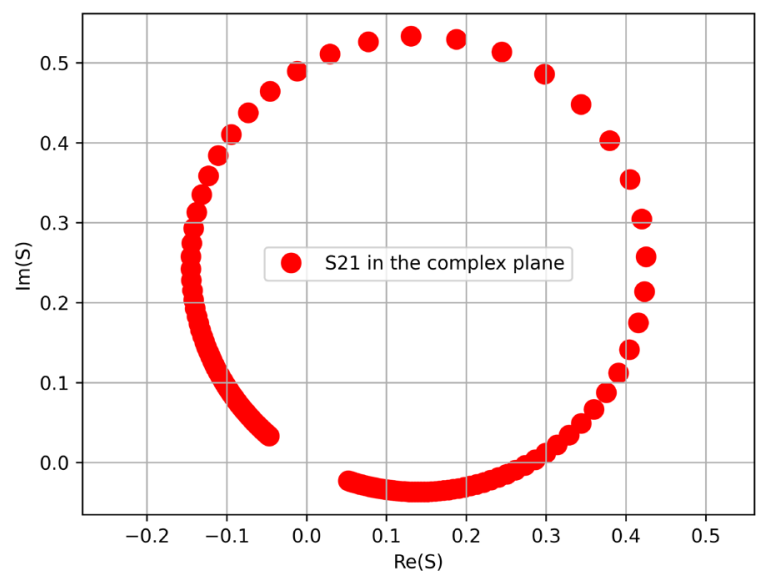

Fig. 2. Distribution of $S_{21}$ measurement points in the complex plane. The linear frequency sweep contains 201 points, is centered at the resonant frequency and has a span equal to 10 times the $3 \mathrm{~dB}$ bandwidth. Note that the density of points is maximum at the edges of the trace.

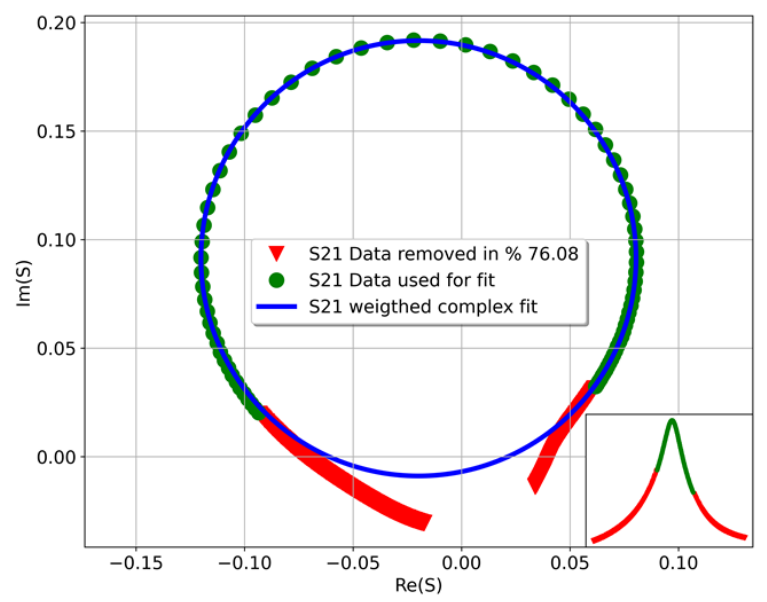

Fig. 3. $S_{21}$ distribution in the complex plane obtained from an uncalibrated measurement of a $27 \mathrm{GHz}$ dielectric resonator processed with the automatic outlier removal procedure $(T h=10)$. Red triangles: points rejected; green circles: points used in the fit; blue trace: fitted response. For a better view only every fifth point is displayed. The inset at the bottom right corner corresponds to $\left|S_{21}(f)\right|$. If no points are removed, resulting $Q_{0}$ diverges by $19 \%$ because the least-squares algorithm is affected by the distorted portion of the trace.

Equation (3) is derived from circuit analysis of a lumpedelement resonator [11],[14],[16]-[18]. It can be rewritten as:

$$
F+2 j Q_{L} G \delta_{i}-2 j Q_{L} S_{i}^{\prime}\left(\delta_{i}-\delta_{e}\right)=S_{i}^{\prime},
$$

where $F=K+G-2 j Q_{L} G \delta_{e}$ is a complex fitting parameter. Equation (5) can be split into its real and imaginary parts:

$F_{R E}-2 Q_{L} G_{I M} \delta_{i}-2 Q_{L} \delta_{e} \operatorname{Im}\left[S_{i}^{\prime}\right]+2 Q_{L} \operatorname{Im}\left[S_{i}^{\prime}\right] \delta_{i}=\operatorname{Re}\left[S_{i}^{\prime}\right]$

$F_{I M}+2 Q_{L} G_{R E} \delta_{i}+2 Q_{L} \delta_{e} \operatorname{Re}\left[S_{i}^{\prime}\right]-2 Q_{L} \operatorname{Re}\left[S_{i}^{\prime}\right] \delta_{i}=\operatorname{Im}\left[S_{i}^{\prime}\right],(6)$

and be written as a system of linear equations:

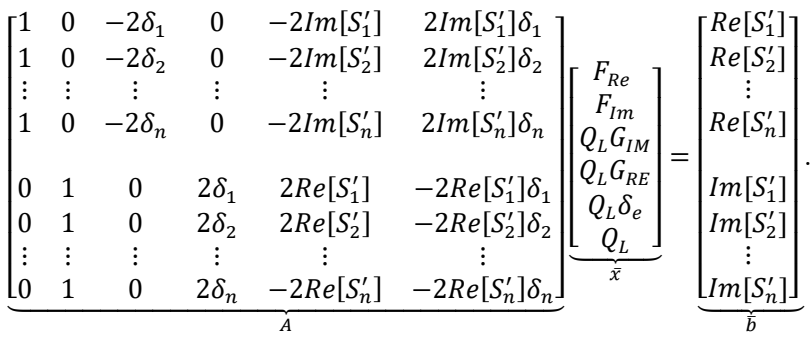

Here, $F_{R e}=\operatorname{Re}[F], F_{I m}=\operatorname{Im}[F], G_{R e}=\operatorname{Re}[G]$, and $G_{I m}=$ $\operatorname{Im}[G]$. Equation (7) is an overdetermined system of equations having 6 real unknowns and twice as many equations as frequency points in the measurement set $(2 n)$. It can be posed in matrix form as $A \bar{x} \simeq \bar{b}$, being $A$ a $2 n \times 6$ matrix and $\bar{x}, \bar{b}$ column vectors of 6 and $2 n$ components respectively. The values of the 6 unknowns in $\bar{x}$ that minimize the least-squares error between the left- and right-hand sides of (7) can be readily found by transforming it into the normal system of equations $A^{T} A \bar{x}=A^{T} \bar{b}$ which has 6 equations and 6 unknowns [19]. However, instead of solving the normal equations using standard routines, we solve the overdetermined system in (7) using Moore-Penrose inverse routines, which are better suited to perform the least-squares approximation [19]. Note that, once the least-squares fit in (7) is performed, we obtain the loaded quality factor $Q_{L}$ and an update of the resonant frequency estimation $f_{0}^{\prime}=f_{0}\left(1+\delta_{e}\right)$. This non-iterative procedure improves on previous works [20],[21] which do a similar complex fit but based on iterative methods (LevenbergMarquardt) that require an initial guess and may fail to give a correct result if they converge on a local minimum of the error function. In our procedure, iteration is reserved for the outlier removal described below.

\section{E. Weights and Outlier Removal}

Fig. 2 shows a typical distribution of $S_{21}$ measurement points along a resonance circle in the complex plane. The linear frequency sweep produced by most network analyzers results in a distribution of points whose density is minimum at resonance and maximum at the edges of the trace, close to the origin of the complex plane. Any least-squares algorithm will tend to prioritize the edges close to the origin which, in turn, are the parts of the trace with the worst signal to noise ratio and most vulnerable to be distorted due to imperfections in the device and measurement setup. To illustrate this, Fig. 3 shows an example of an experimentally determined $S_{21}$ trace showing distortion at the tails of the trace. This type of distortion can arise by multiple causes, among others: nearby resonant modes, frequency-dependent cross-talk, frequency-dependent loss or dispersion in the test-set and cables between the VNA and the resonator. It is apparent from this figure that distortion, combined with the inhomogeneous distribution of measurement points will lead to erroneous results to any least-squares fitting software.

Applying weights to (7) is an effective solution to reduce the influence of the trace edges to the overall fitting error. One simple solution is to multiply the first $n$ equations by $\operatorname{Re}\left[S_{21}^{\prime}\right]$ and the following ones by $\operatorname{Im}\left[S_{21}^{\prime}\right]$. This gives good results with $S_{21}$ traces having little or no distortion. However, for traces 


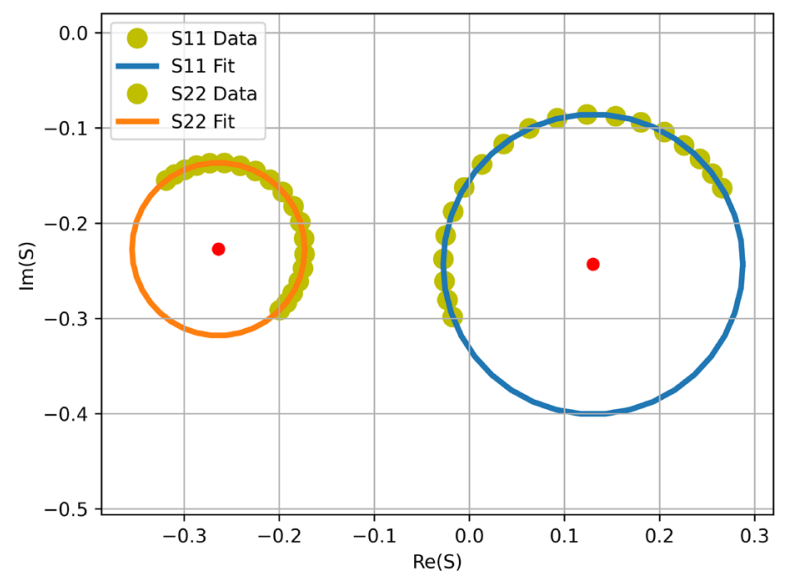

Fig. 4. Distribution of input and output reflection coefficient in the complex plane. Green points: measured data within $3 \mathrm{~dB}$ bandwidth. Solid lines: leastsquares circle fits. Red points: fitted circle centers.

with significant distortion such as the one in Fig. 3, removal of the outlier points is needed.

Our algorithm performs an adaptive outlier removal. Once the weighted least-squares fit on $S_{i}^{\prime}$ is performed, the algorithm computes the fitting error below:

$$
\epsilon_{i}=\left|\frac{1}{\left(S_{i}^{\prime}-G\right)}-\left(\frac{K}{1+2 j Q_{L}\left(\delta_{i}-\delta_{e}\right)}\right)^{-1}\right|
$$

which tends to magnify errors at frequencies further away from resonance and thus, more prompt to be affected by distortion. The point with the highest error $\epsilon_{\max }$ is identified and its error is compared to a threshold:

$$
\epsilon_{\max }<\frac{1}{T h} \cdot \frac{1}{|K|}
$$

where $K$ is the fitting parameter appearing in (3) and $T h$ is a threshold scaling parameter. If the condition posed in (9) is not satisfied, the corresponding measurement point with the highest fitting error is removed from the data set and the least-squares fit is re-calculated. This recursive process is iteratively repeated until (9) is finally satisfied. Note that both $Q_{L}$ and $f_{0}$ are recursively updated in the outlier removal process. Fig. 3 shows how effective this procedure is in removing distorted points from both ends of the $S_{21}$ trace and fitting to the undistorted portion of the trace.

Fixing the value of $T h$ is a compromise between sensitivity to noise and sensitivity to distortion. On the one hand, the larger $T h$, the more data will be removed, and the fitting will be unaffected by large distortion in the trace tails. On the other hand, by using a few data points in the least squares fit (7), the results will be more sensitive to noise in $S_{21}$. We performed an extensive study taking into account this compromise and have set $T h=10$ as a good value for this parameter.

\section{F. Reflection Response}

The reflection response is used to calculate the input and output coupling factors $\left(\beta_{1}, \beta_{2}\right)$ needed to determine the unloaded quality factor $\left(Q_{0}\right)$ from the loaded one $\left(Q_{L}\right)$ through $Q_{0}=\left(1+\beta_{1}+\beta_{2}\right) Q_{L}$.

The coupling factors can be calculated from the magnitudes of $S_{11}$ and $S_{22}$ at resonance $\left(\left|S_{11 \_0}\right|,\left|S_{22 \_0}\right|\right)[4]$ :

$$
\beta_{1}=\frac{1-\left|S_{11 \_0}\right|}{\left|S_{11 \_0}\right|+\left|S_{22_{-} 0}\right|}, \beta_{2}=\frac{1-\left|S_{22 \_0}\right|}{\left|S_{11 \_0}\right|+\left|S_{22 \_}\right|} .
$$

To accurately calculate $\left|S_{11_{\_} 0}\right|,\left|S_{22 \_0}\right|$ taking into account the loss between the calibration plane and the resonator, the algorithm performs geometric least-squares circle fits of $S_{11}(f)$ and $S_{22}(f)$ close to resonance, and determines their complex centers $C_{11}, C_{22}$ and radii $r_{11}, r_{22}$ [20]:

$$
\begin{aligned}
& \underbrace{\left[\begin{array}{ccc}
\operatorname{Re}\left[S_{m m}\right]_{1} & \operatorname{Im}\left[S_{m m}\right]_{1} & 1 \\
\operatorname{Re}\left[S_{m m}\right]_{2} & \operatorname{Im}\left[S_{m m}\right]_{2} & 1 \\
\vdots & \vdots & \vdots \\
\operatorname{Re}\left[S_{m m}\right]_{n} & \operatorname{Im}\left[S_{m m}\right]_{n} & 1
\end{array}\right]}_{M} \underbrace{\left[\begin{array}{l}
a_{1} \\
a_{2} \\
a_{3}
\end{array}\right]}_{a} \approx \underbrace{\left[\begin{array}{c}
-\left|S_{m m}\right|_{1}^{2} \\
-\left|S_{m m}\right|_{2}^{2} \\
\vdots \\
-\left|S_{m m}\right|_{n}^{2}
\end{array}\right]}_{P}, \\
& C_{m m}=\left(-\frac{a_{1}}{2},-\frac{a_{2}}{2}\right), r_{m m}=\sqrt{\frac{1}{4}\left(a_{1}^{2}+a_{2}^{2}\right)-a_{3}},
\end{aligned}
$$

where the index is either $m=1$ or 2 , and $a_{1}, a_{2}$ and $a_{3}$ are the fitting parameters to be found using the pseudo-inverse technique to minimize the least square error in (11). Since, in the absence of loss, these circles should be tangent to the unit circle [11], [21], the input and output return loss at resonance are respectively $\left|C_{11}+r_{11} \cdot C_{11} /\right| C_{11}||^{-1}$ and $\left|C_{22}+r_{22} \cdot C_{22}\right|$ $\left.\left|C_{22}\right|\right|^{-1}$. The minima in $\left|S_{11}(f)\right|,\left|S_{22}(f)\right|$ correcting for this loss are:

$$
\begin{gathered}
\left|S_{11 \_0}\right|=\left|\frac{C_{11}-r_{11} \cdot C_{11} /\left|C_{11}\right|}{C_{11}+r_{11} \cdot C_{11} /\left|C_{11}\right|}\right|=\frac{|| C_{11}\left|-r_{11}\right|}{\left|C_{11}\right|+r_{11}}, \\
\left|S_{22 \_0}\right|=\frac{|| C_{22}\left|-r_{22}\right|}{\left|C_{22}\right|+r_{22}} .
\end{gathered}
$$

Note that by using (13) and (14), the loss is evaluated at the resonant frequency and the algorithm removes the effect of any frequency-dependent loss between the calibration planes and the resonator ports. Similarly to (7), the least-squares fit of the resonance circles in (11) is done using Moore-Penrose inverse routines [20]. Only the points for which $\left|Q_{L} \delta_{i}\right| \leq 1 / 2$ are used for the fitting. This covers half the perimeter of the resonance circle and, at the same time, avoids using off-resonance measurement points in the circle fit. Fig. 4 shows an example for this type of fit.

Nevertheless, at very low coupling $\left(\left|S_{21}\right|\right.$ maximum below $-50 \mathrm{~dB})$ the resonance circles in $S_{11}(f)$ and $S_{22}(f)$ are very small and the geometric least-squares circle fit does not work properly. In this case, the accuracy in the determination of coupling factors has no practical effect. However, instead of neglecting the coupling coefficients completely, we apply (10): $\left|S_{11_{1} 0}\right|$ and $\left|S_{22 \_0}\right|$ are taken from the values of $S_{11}, S_{22}$ corresponding to the frequency at which $\left|S_{21}^{\prime}\right|$ is maximum and compensated for losses (such as those generated by transmission lines between the calibration plane and the 


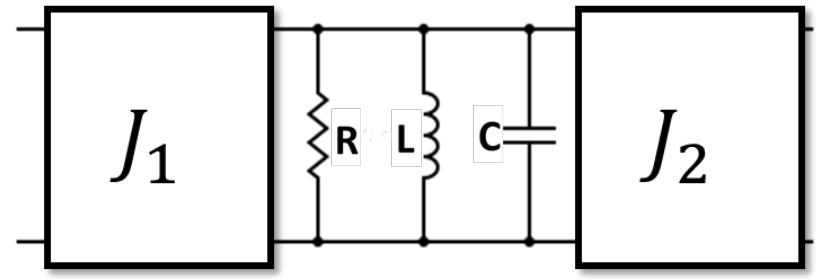

Fig. 5. Equivalent circuit of a two-port resonator

resonator), which are estimated from the off-resonance values of $\left|S_{11}\right|$ and $\left|S_{22}\right|$ (which should be $0 \mathrm{~dB}$ in absence of loss).

To estimate the off-resonance values of $\left|S_{11}\right|$ and $\left|S_{22}\right|$, the algorithm averages its values in the $10 \%$ upper and lower fractions of the frequency span (as in Sect. II.B).

\section{Algorithm Performance AsSESSMENT}

Two types of performance assessment have been carried out. On the one hand, an experimental assessment verifies the consistency of the algorithm results obtained from measurements performed under a variety of conditions. On the other hand, a simulated assessment verifies the algorithm with S-parameter files obtained from circuit analysis whose couplings, quality factors and resonant frequencies are known and can be compared against the algorithm's results.

To assess the algorithm's performance with symmetrical transmission responses, we have used a two-port, iris-coupled WR-340 waveguide resonator. The resonator has a $196 \mathrm{~mm}$ long waveguide with two $25 \mathrm{~mm}$ circular irises at both ends and waveguide to coaxial transitions. By using the first four modes ( $\mathrm{TE}_{10 \mathrm{p}}, p=1 . .4$ ), we obtain coupling values ranging from 0.009 to 0.64 (see Table I). To measure the resonator, we used a Rohde-Schwarz ZNA26 network analyzer having GORE VNA cables (FB0HA0HB0250, typical stability $0.01 \mathrm{~dB} 2.0^{\circ}$, maximum $0.08 \mathrm{~dB} 3.9^{\circ}$ ) with a coaxial calibration using the electronic calibration module of the unit. Torque wrenches were used to fasten the connectors. A GPS-disciplined $10 \mathrm{MHz}$ external frequency reference providing $0.01 \mathrm{ppm}$ frequency accuracy was used. A single, 16001 point calibration was performed to cover the four modes. When measuring a specific mode, we reduced the number of points to 201 and changed the rest of frequency sweep parameters to match those described in Sect. II.A.

\section{A. Uncertainty in Equivalent Circuit Fitting and VNA Calibration - Waveguide Resonator.}

An equivalent circuit (Fig. 5) was derived for each mode of the WR-340 waveguide resonator using $f_{0}, Q_{0}, \beta_{1}$ and $\beta_{2}$ extracted from calibrated measurements with $100 \mathrm{~Hz}$ IF bandwidth. The values of the resistance $(R)$, inductance $(L)$, capacitance $(C)$, and admittances transformation constant $J_{1}, J_{2}$ for the equivalent circuit were found using standard resonator formulas for $f_{0}$ and $Q_{0}$, and the equation that relates coupling values with the admittance transformation constant: $J_{i}=$ $\sqrt{\beta_{i} /\left(Z_{0} R\right)}$. The resulting equivalent circuits were analyzed using the commercial software (ADS) [22] and S-parameters were produced to test ability of our code to fit data not subject to noise or distortion.
TABLE I

RESONATOR PARAMETERS. ESTIMATED UNCERTAINTIES DUE TO CALIBRATION AND EQUIVALENT CIRCUIT FITTING

\begin{tabular}{lcccc}
\hline \hline & $\mathrm{TE}_{101}$ & $\mathrm{TE}_{102}$ & $\mathrm{TE}_{103}$ & $\mathrm{TE}_{104}$ \\
\hline$\left|S_{21}\left(f_{0}\right)\right|_{V N A}$ & $-33.6 \mathrm{~dB}$ & $-11.8 \mathrm{~dB}$ & $-6.4 \mathrm{~dB}$ & $-5.6 \mathrm{~dB}$ \\
$f_{0_{-} V N A}(G H z)$ & 1.900636 & 2.301379 & 2.845639 & 3.465098 \\
$\left(\Delta f_{0} / f_{0}\right)_{E Q}$ & $2.63 \mathrm{E}-11$ & $9.16 \mathrm{E}-11$ & $8.21 \mathrm{E}-12$ & $1.30 \mathrm{E}-10$ \\
$\left(\Delta f_{0} / f_{0}\right)_{C A}$ & $2.40 \mathrm{E}-05$ & $2.05 \mathrm{E}-05$ & $1.68 \mathrm{E}-05$ & $1.40 \mathrm{E}-05$ \\
$\left(10 Q_{L}\right)_{V N A}^{-1}$ & $1.22 \mathrm{E}-05$ & $2.12 \mathrm{E}-05$ & $3.48 \mathrm{E}-05$ & $3.65 \mathrm{E}-05$ \\
$Q_{L_{-} V N A}$ & $8,184.99$ & $4,717.25$ & $2,876.27$ & $2,738.00$ \\
$\left(\Delta Q_{L} / Q_{L}\right)_{E Q}$ & $2.47 \mathrm{E}-06$ & $5.48 \mathrm{E}-07$ & $1.96 \mathrm{E}-08$ & $2.82 \mathrm{E}-07$ \\
$\left(\Delta Q_{L} / Q_{L}\right)_{C A}$ & $1.80 \mathrm{E}-03$ & $8.51 \mathrm{E}-04$ & $2.33 \mathrm{E}-04$ & $5.53 \mathrm{E}-04$ \\
$\beta_{1} V N A$ & 0.0125 & 0.1472 & 0.4347 & 0.6443 \\
$\left(\Delta \beta_{1} / \beta_{1}\right)_{E Q}$ & $1.24 \mathrm{E}-02$ & $4.59 \mathrm{E}-12$ & $9.81 \mathrm{E}-10$ & $7.30 \mathrm{E}-10$ \\
$\left(\Delta \beta_{1} / \beta_{1}\right)_{C A}$ & $5.16 \mathrm{E}-04$ & $2.73 \mathrm{E}-03$ & $9.37 \mathrm{E}-04$ & $1.68 \mathrm{E}-03$ \\
$\beta_{2} V N A$ & 0.0093 & 0.1808 & 0.4633 & 0.4736 \\
$\left(\Delta \beta_{2} / \beta_{2}\right)_{E Q}$ & $1.25 \mathrm{E}-02$ & $3.46 \mathrm{E}-12$ & $1.85 \mathrm{E}-10$ & $3.27 \mathrm{E}-10$ \\
$\left(\Delta \beta_{2} / \beta_{2}\right)_{C A}$ & $3.27 \mathrm{E}-03$ & $3.34 \mathrm{E}-04$ & $8.80 \mathrm{E}-04$ & $7.72 \mathrm{E}-04$ \\
$Q_{0} V N A$ & $8,363.50$ & $6,264.57$ & $5,459.19$ & $5,798.84$ \\
$\left(\Delta Q_{0} / Q_{0}\right)_{E Q}$ & $2.69 \mathrm{E}-04$ & $5.48 \mathrm{E}-07$ & $1.93 \mathrm{E}-08$ & $2.81 \mathrm{E}-07$ \\
$\left(\Delta Q_{0} / Q_{0}\right)_{C A}$ & $1.84 \mathrm{E}-03$ & $1.20 \mathrm{E}-03$ & $6.63 \mathrm{E}-04$ & $1.24 \mathrm{E}-03$ \\
\hline \hline
\end{tabular}

Resonator parameters and their relative variations. Subscripts EQ indicate relative differences of fitted parameters with respect to nominal equivalent circuit values. Subscripts CA indicate differences obtained fitting calibrated vs. uncalibrated VNA measurements.

TABLE II

Relative Differences BetweEn Measurement Setups

\begin{tabular}{lcccc}
\hline \hline & $\mathrm{TE}_{101}$ & $\mathrm{TE}_{102}$ & $\mathrm{TE}_{103}$ & $\mathrm{TE}_{104}$ \\
\hline$\left(\Delta Q_{0} / Q_{0}\right)$ & $3.77 \mathrm{E}-03$ & $2.72 \mathrm{E}-02$ & $1.38 \mathrm{E}-02$ & $5.35 \mathrm{E}-03$ \\
$\left(\Delta f_{0} / f_{0}\right)$ & $3.64 \mathrm{E}-05$ & $2.99 \mathrm{E}-05$ & $2.59 \mathrm{E}-05$ & $2.26 \mathrm{E}-05$ \\
$\left(10 Q_{L}\right)^{-1}$ & $1.22 \mathrm{E}-05$ & $2.10 \mathrm{E}-05$ & $3.51 \mathrm{E}-05$ & $3.72 \mathrm{E}-05$ \\
\hline \hline
\end{tabular}

Relative differences obtained by fitting VNA parameters with the two measurement setups used. The factor $\left(10 Q_{L}\right)^{-1}$ is included for comparison with relative frequency differences.

Table I summarizes the results. It lists, for the four modes, the resonator parameters obtained from VNA measurements at $100 \mathrm{~Hz}$ IF bandwidth (see VNA subscripts in table). It also shows the relative differences between the resonator parameters extracted from the equivalent circuit S-parameters, and those corresponding to the component values in the circuit (see EQ subscripts in the table). A third type of relative difference shown in Table I corresponds to the difference between resonator parameters obtained from calibrated VNA data and those obtained without calibration (see CA subscripts in table). Finally, the factor $1 /\left(10 Q_{L}\right)$ is also listed in Table I for comparison with the relative differences in $f_{0}$.

\section{B. Uncertainty due to Noise - Waveguide Resonator}

To assess the uncertainty due to instrument noise, we performed VNA measurements at IF bandwidths ranging from $100 \mathrm{~Hz}$ to $1 \mathrm{MHz}$. Once all four modes were measured, one of the cables was disconnected from the resonator to isolate the two ports of the analyzer. In these conditions, the noise floor $(N F)$ of the analyzer was estimated by averaging the noise variance $(\sigma)$ in the 201 points of the frequency sweep and converting it to $\mathrm{dB}(N F=20 \log \sigma)$. We have checked that, as expected, the noise floor decreases by $10 \mathrm{~dB}$ for every tenfold reduction in the IF bandwidth and that the resonance peak in the mode with the weakest coupling ( $\mathrm{TE}_{101}$ ) was $30 \mathrm{~dB}$ above the noise floor for a $1 \mathrm{MHz}$ IF bandwidth. Since the IF bandwidth can be reduced several orders of magnitude without practical 


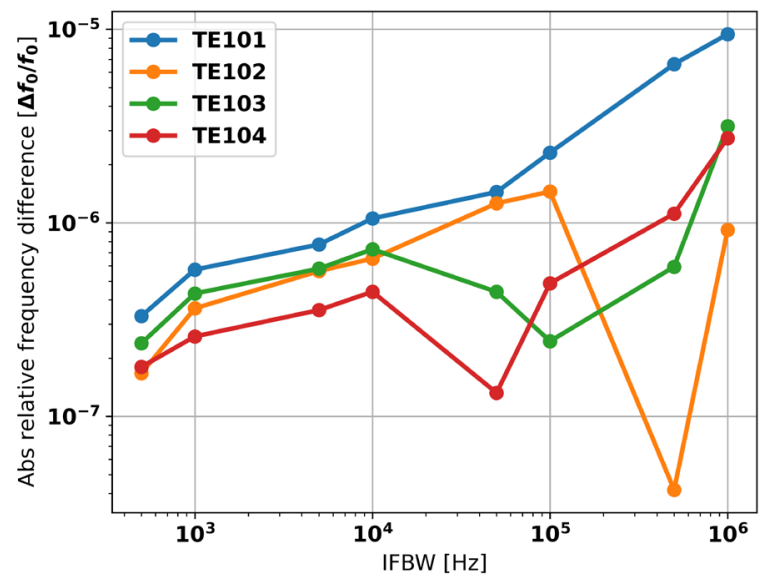

Fig. 6. Absolute relative frequency differences taking the $100 \mathrm{~Hz}$ IF bandwidth results as reference values.

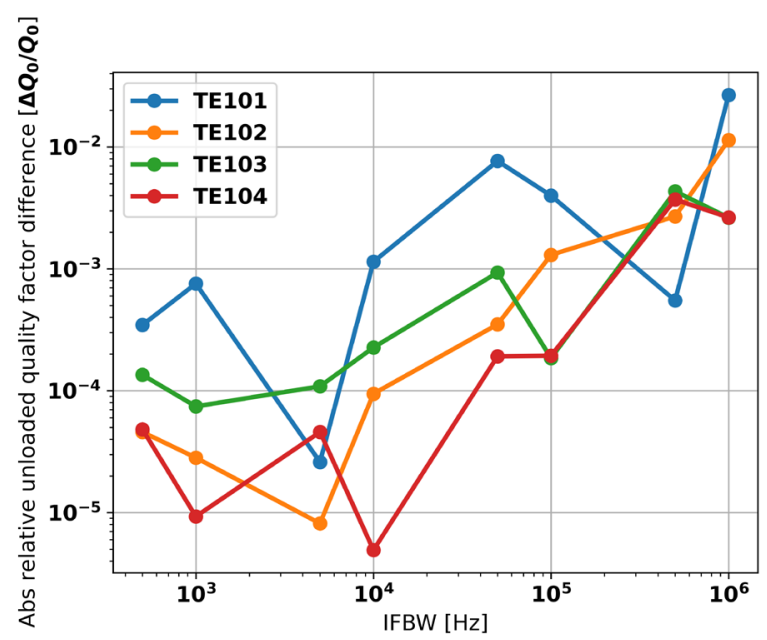

Fig. 7. Absolute relative unloaded quality factor differences taking the $100 \mathrm{~Hz}$ IF bandwidth results as reference values.

consequences, it is possible to measure resonators with much weaker coupling than the $\mathrm{TE}_{101}$ mode.

Fig. 6 and 7 show the relative differences for $f_{0}$ and $Q_{0}$ with respect to their reference values obtained from VNA Sparameters at $100 \mathrm{~Hz}$ IF bandwidth. Overall, the uncertainty due to instrument noise is lower than that caused by other factors in the extreme case of $1 \mathrm{MHz}$ IF bandwidth, the relative differences are $9 \cdot 10^{-6}$ for $f_{0}$ and $2.7 \%$ for $Q_{0}$ in the $\mathrm{TE}_{101}$ mode. Note that, in normal practice, IF bandwidths are at most $10 \mathrm{kHz}$ and the relative differences are much smaller.

\section{Uncertainty due to Equipment - Waveguide Resonator}

We have compared the measurements above with another set of measurements made with a Keysight PNA-X VNA. The waveguide resonator was placed within reach of both analyzers and not moved during the tests. This second set of measurements was made with $1 \mathrm{~m}$ long UNIRTest-W27 cables having $0.05 \mathrm{~dB}$ and $\pm 4.5^{\circ}$ mechanical stability at $40 \mathrm{GHz}$. Calibration and measurement followed the procedures described above. Table II shows the relative difference for $f_{0}$ and $Q_{0}$ obtained with the two VNAs at $100 \mathrm{~Hz}$ IF bandwidth.

\section{Least Squares Fit-Waveguide Resonator}

As discussed in Sect. II.D, the least-squares fit in (7) can be done using the Moore-Penrose inverse or by solving the normal equations. The Moore-Penrose inverse is generally recommended over the normal equations because of lower matrix condition numbers in the matrix computations [19]. We have evaluated both alternatives using the measured Sparameter data of the four modes at $100 \mathrm{~Hz}$ IF bandwidth. The maximum relative differences between the two methods in the four modes is $2.4 \cdot 10^{-15}$ for $Q_{0}$ and below $1 \cdot 10^{-15}$ for $f_{0}$. These values are much smaller than those of the preceding subsections and indicate that matrix computation uncertainties are negligible.

\section{E. Fitting Fano Responses - Rutile Dielectric Resonator}

To test the algorithm against Fano responses, we have compared different modes in a rutile dielectric resonator [23]. In this cavity, the $\mathrm{TE}_{012}$ mode is Lorentzian and the $\mathrm{TE}_{013}$ follows a Fano distribution (Fig. 1). The unloaded quality factor in the resonator depends on the surface resistance of the metal endplates $\left(R_{S 1}\right.$ and $\left.R_{S 2}\right)$, lateral walls $\left(R_{L}\right)$ and dielectric loss $(p \cdot \tan \delta)$ through the equation below [12]:

$$
\frac{1}{Q_{0}}=\frac{R_{S 1}+R_{S 2}}{G_{S}}+\frac{R_{L}}{G_{L}}+p \cdot \tan \delta,
$$

where $G_{S}, G_{L}$ and $p$ are geometric factors that can be calculated from the field distribution [4]. We have measured the quality factors of the resonator at both modes using a round robin combination of cooper and beryllium cooper endplates. Using

TABLE III

RELATIVE DIFFERENCE IN THE UNLOADED QUALITY FACTOR FOR COMBINATIONS OF COPPER (CU) AND COPPER-BERYLLIUM (CUBE)

\begin{tabular}{ll}
\hline \hline $\begin{array}{c}\text { Metal } \\
\text { endplates }\end{array}$ & Relative difference in $Q_{0}$ \\
\hline $\mathrm{Cu} \mathrm{Cu}$ & $2.66 \mathrm{E}-03$ \\
$\mathrm{Cu} \mathrm{CuBe}$ & $2.73 \mathrm{E}-03$ \\
$\mathrm{CuBe} \mathrm{CuBe}$ & $4.25 \mathrm{E}-03$ \\
\hline \hline
\end{tabular}

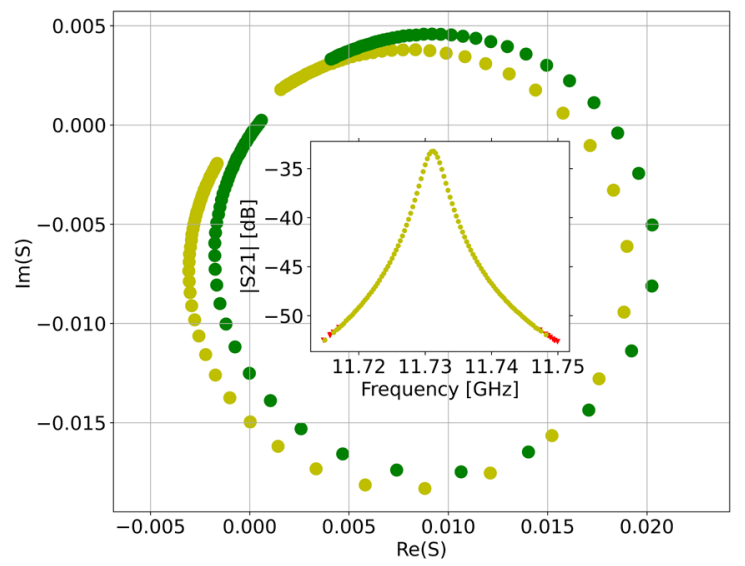

Fig. 8. Postprocessed Fano $S_{21}$ distribution in the complex plane obtained from the multi mode dielectric resonator. Shift with respect to the origin of the complex plane is indicated by showing the original measurement data. The inset at the center corresponds to $\left|S_{21}^{\prime}(f)\right|$. 


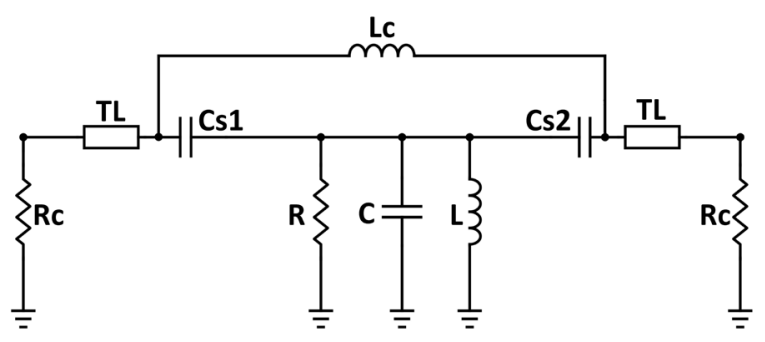

Fig. 9. Equivalent circuit used for verification. It includes transmission lines and a feedthrough inductor to simulate stray coupling between resonator ports in sets 3 and 4 . In these sets, the transmission response was asymmetrical with the lower frequencies having higher transmission coefficients. A feedthrough capacitor would have been used instead of the inductor for the opposite type of asymmetry.

the procedure described in [23], we have determined $R_{S 1}, R_{S 2}$, $R_{L}$, and $p \cdot \tan \delta$ for the $\mathrm{TE}_{012}$ mode and $p \cdot \tan \delta$ for the $\mathrm{TE}_{013}$ mode. Then we have predicted $Q_{0}$ for the $\mathrm{TE}_{013}$ mode assuming that surface resistance scales with the square root of frequency. Table III shows the relative differences in $Q_{0}$ for the various combinations of metal endplates. By comparison, the relative difference obtained when calculating $Q_{L}$ in the $\mathrm{TE}_{013}$ mode in Fig. 1 with our algorithm and with the $3-\mathrm{dB}$ method is $3.5 \%$, about an order of magnitude larger than those in Table III.

Fig. 8 shows the output from our code, showing that the simple shift performed in (2) converts $\left[S_{21}\right\rceil$ from a Fano distribution (Fig. 1) to a quasi-Lorentzian one (inset Fig. 8).

\section{F. Simulated Assessment}

For the simulated assessment, we used the commercial software ADS [22] to fit equivalent circuits (Fig. 9) to Sparameter data from four different measurements (Fig. 10 to 13) taken on different resonators and different samples representing extreme situations that would make fitting difficult, such as low quality factor or asymmetry in the transmission response $\left(\left|S_{21}(f)\right|\right)$. Details on the data sets follow:

- Set 1: Large $Q\left(Q_{0}=64110\right)$. Low-moderate coupling: $\left(\beta_{1} \approx 0.05, \beta_{2} \approx 0.1, \max \left|S_{21}(f)\right|=-21.1 \mathrm{~dB}\right)$.

Slope in the off-resonance $\left|S_{11}(f)\right|$. Transmission response $\left|S_{21}(f)\right|$ symmetrical with respect to the maximum. Large frequency span of 73 times the $3 \mathrm{~dB}$ bandwidth. Fitting challenge: only a few points are in the $3 \mathrm{~dB}$ bandwidth region.

- $\quad$ Set 2: Very large $Q\left(Q_{0}=204368\right)$. High asymmetric coupling: $\quad\left(\beta_{1}=0.85, \beta_{2}=0.48, \max \left|S_{21}(f)\right|=\right.$ $-7.9 \mathrm{~dB})$. No off-resonance slope in $\left|S_{11}(f)\right|$ or $\left|S_{22}(f)\right|$. Transmission response $\left|S_{21}(f)\right|$ symmetrical with respect to the maximum. Regular span of 10 times the $3 \mathrm{~dB}$ bandwidth. Fitting challenge: the coupling is very asymmetric and considered as high ( $\beta_{1}$ close to 1$)$ which usually leads to high uncertainty in $Q_{0}$.

- Set 3: Low $Q\left(Q_{0}=2635\right)$. Low, asymmetric coupling: $\left(\beta_{1} \approx 0.002, \beta_{2}<0.001, \max \left|S_{21}(f)\right|=-55.0 \mathrm{~dB}\right)$. Off-resonance slope in $\left|S_{11}(f)\right|$ and $\left|S_{22}(f)\right|$. Asymmetry in $\left|S_{21}(f)\right|$. Regular span of 10 times the

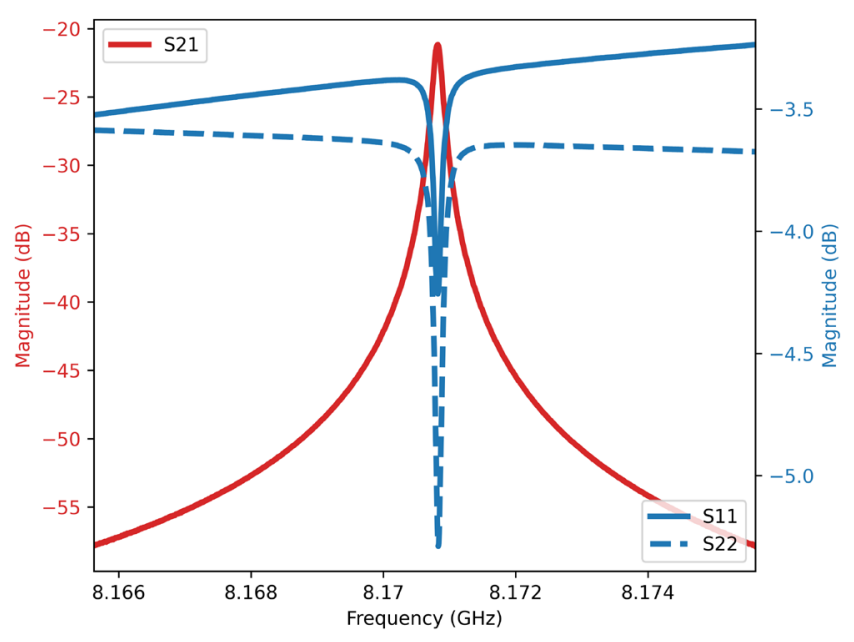

Fig. 10. Magnitude of measured S-parameters of Set 1.

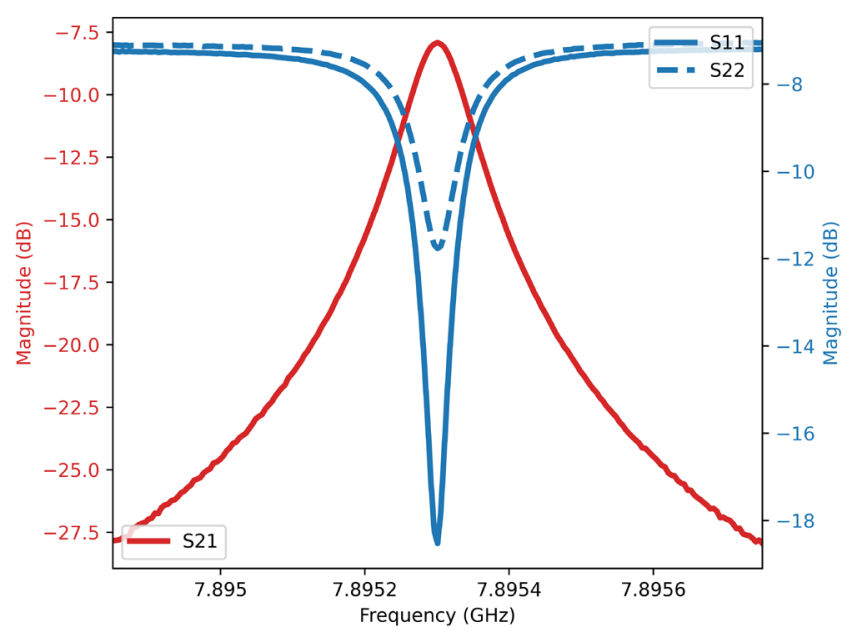

Fig. 11. Magnitude of measured S-parameters of Set 2.

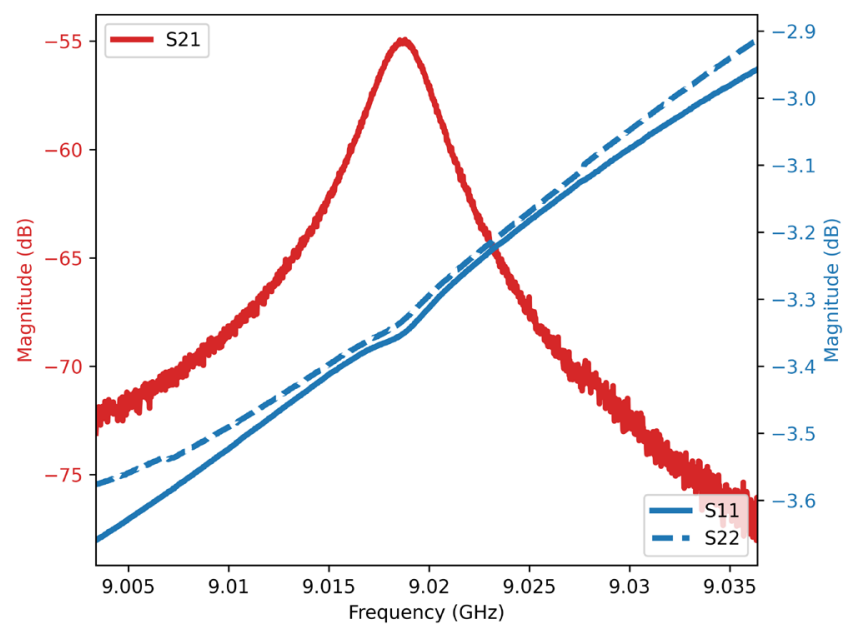

Fig. 12. Magnitude of measured S-parameters of Set 3.

$3 \mathrm{~dB}$ bandwidth. Fitting challenge: low coupling and low quality factor results in high measurement noise.

- Set 4: Very low $Q \quad\left(Q_{0}=475\right)$. Low, symmetric coupling: $\left(\beta_{1}, \beta_{2} \approx 0.02, \max \left|S_{21}(f)\right|=-34.4 \mathrm{~dB}\right)$. Off-resonance slope in $\left|S_{11}(f)\right|$ and $\left|S_{22}(f)\right|$. 


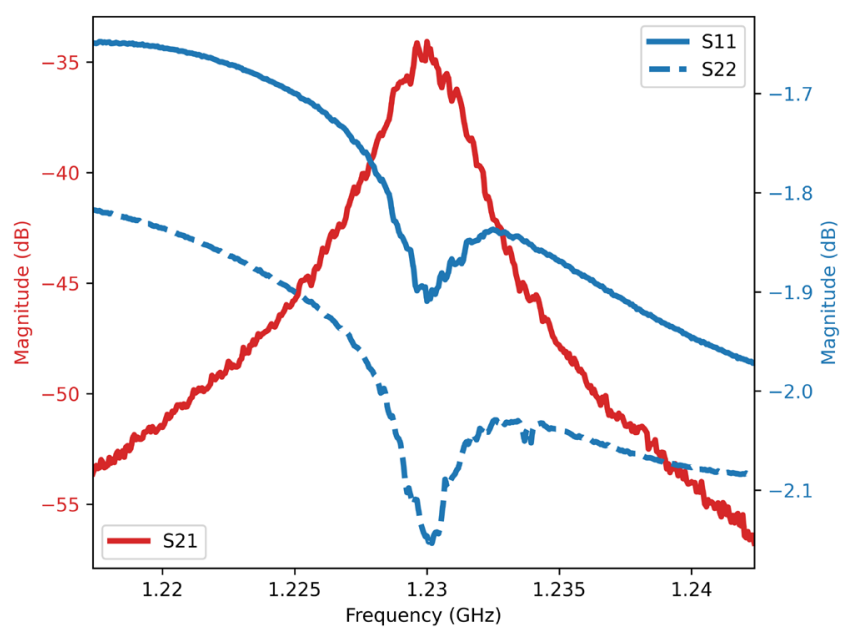

Fig. 13. Magnitude of measured S-parameters of Set 4.

Asymmetry in $\left|S_{21}(f)\right|$. Regular span of 10 times the $3 \mathrm{~dB}$ bandwidth. Fitting challenge: off-resonance slope in $\left|S_{11}(f)\right|$ and $\left|S_{22}(f)\right|$ generates inaccuracy in the calculation of coupling coefficients.

Each of the data sets above was fitted to two different equivalent circuits: one following Fig. 9 which included transmission lines between the S-parameter ports and the resonator, and another version not including these lines. This was done to test the effect of transmission lines in the algorithm's fitting accuracy [14].

The S-parameters of equivalent circuits were obtained through circuit analysis and then perturbed to simulate VNA noise. To realistically assess VNA noise, we assumed it behaves as band-pass Gaussian noise [24], [25]. In this type of noise, the variance in the real and imaginary part is equal to the overall variance in the noise envelope. Accordingly, the standard deviation used in the statistical analysis is related to the VNA noise floor $(N F)$ through:

$$
\sigma=10^{N F(d B) / 20}
$$

The real and imaginary part of the S-parameter data obtained from circuit analysis was perturbed at each frequency point by adding random Gaussian noise whose variance is adjusted to a specific $N F$ ranging from -100 to $-60 \mathrm{~dB}$. For the statistical analyses, this process is done 20 times per each value of $N F$ assessed, so each unperturbed S-parameter file produces 20 perturbed files per each value of instrument noise floor. The perturbed S-parameter files are then fed to the fitting algorithm and the resulting values of $Q_{0}, f_{0}$ are compared against the nominal values from the equivalent circuit. Every point in Fig. 14 and 15 is calculated by averaging the resulting 20 absolute relative differences with respect to the nominal value.

Fig. 14 shows the relative uncertainty in $Q_{0}$ for Set 1 versus simulated VNA noise floor. The effect of the transmission lines is to set a residual uncertainty (close to $0.4 \%$ ) that cannot be removed regardless of the efforts made to reduce noise (by reducing IF bandwidth, increasing the number of points in the trace or averaging over several sweeps). Fig. 14 also shows that

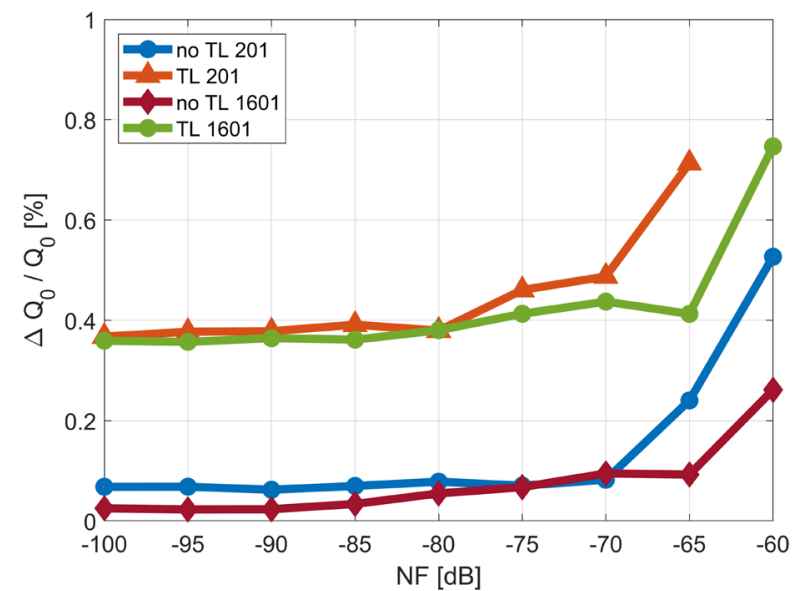

Fig. 14. Relative uncertainty as a function of simulated VNA noise floor in data set 1. TL (insert) refers to the inclusion of $1.5 \mathrm{~m}$ transmission lines in the equivalent circuit used to produce the S-parameter files. The values 201 and 1601 indicate the number of points in the frequency span.

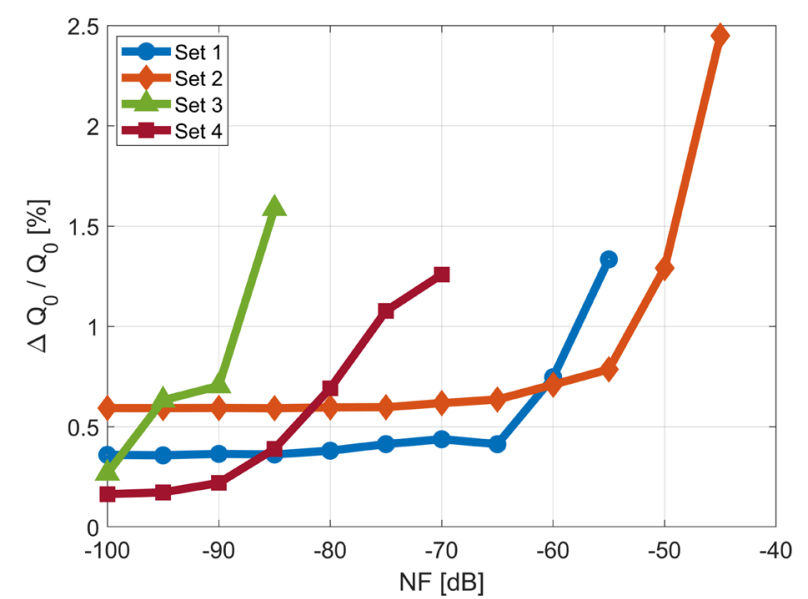

Fig. 15. Relative uncertainty with 1601 points per trace as a function of simulated VNA noise floor for the four sets. The vertical scale has been chosen to show only uncertainties below $2.5 \%$.

there is a threshold in VNA noise that produces a large uncertainty increase (about $-70 \mathrm{~dB}$ in this specific case). The figure also shows that the number of points in the frequency sweep affects the noise threshold but not the residual uncertainty produced by the transmission lines.

Fig. 15 compares the uncertainties in the four data sets produced by the equivalent circuits with transmission lines. The figure shows that, in absence of other sources of uncertainty, it is possible to achieve uncertainties of a fraction of $1 \%$ if the VNA noise is made sufficiently small. Note that hand-held VNAs have noise floors close to $-73 \mathrm{~dB}$ at $1 \mathrm{kHz}$ IF bandwidth, which can be reduced at the expense of longer sweep times, so reaching the low-noise asymptotic uncertainty values in Fig. 14 is achievable in most modern VNAs. Fig. 15 also shows the threshold VNA noise for which the algorithm fails for the various data sets. High $\left|S_{21}(f)\right|$ peaks favor large thresholds.

These results seem to compare favorably with those of other publications. For a resonator having $Q_{0}=10000$ under noiseless conditions, [14] reports a relative difference of $3.3 \%$ when 30 wavelength-long transmission lines are included between the calibration plane and the resonator $\left(\beta_{1}=\beta_{2}=\right.$ 
0.08). These uncertainties should be compared to those in Figs. 10 and 11 for $N F=-100 \mathrm{~dB}$, which are obtained under more unfavorable conditions (including 65 wavelength-long cables). When an uncertainty noise radius of 0.001 is added to the Sparameters in [14], a $0.6 \%$ uncertainty is obtained under moderate coupling $\left(\beta_{1}=\beta_{2}=0.08\right), 401$ trace points, and no transmission lines between the device and the calibration plane, which is similar to the two lower traces in Fig. 14 for the corresponding value of noise floor $(N F=-60 \mathrm{~dB})$. These results also compare well with respect to those reported in [18], where including $\mathrm{a} \sim 1 \mathrm{~m}$ cable between the resonator and the calibration plane results in a $7 \%$ difference in $Q_{0}$.

We have also performed an extensive study on the relative uncertainty in resonance frequency $f_{0}$. We have found that $Q_{0}$ is more sensitive to VNA noise than $f_{0}$. Accordingly, once below the noise thresholds indicated in Fig. 14 and 15, the relative uncertainty in $f_{0}$ has very little sensitivity to noise. Below the noise threshold for $Q_{0}$, the order of magnitude of $\Delta f_{0} / f_{0}$ is about $1 /\left(10 Q_{L}\right)$ regardless of whether we use 201 or 1601 points in the frequency sweep. This is consistent with the data shown in Tables I and II.

\section{CONCLUSIONS}

We have developed an algorithm capable of fitting Lorentzian (symmetrical) and Fano (asymmetrical) responses.

The algorithm can be implemented in a highly automated code, capable of remote web execution, where only a load of the S-parameter file generated by the VNA is needed. This enables its use for non-specialists in microwave measurements and favors the fair comparison of results obtained at different institutions on similar devices. A key technical feature of the algorithm that enables its use for non-specialists is the possibility of achieving good fits without the need of manually removing some of the points in the measurement set. This is achieved through an automatic outlier removal process and a compensation of the effects produced by uneven distribution of measurement points along the resonance circles in the complex plane resulting from the VNA's linear frequency sweep.

The algorithm has proven capable of fitting resonator parameters produced by an ideal equivalent circuit (Fig. 9) with negligible differences with respect to the nominal circuit values.

Uncertainties seem to be dominated by distortion, that is, Sparameters not conforming to the ideal ones resulting from the equivalent circuit. Effects of cabling, connectors, calibration and frequency dependence in coupling networks dominate over those produced by noise in current VNAs using standard IF bandwidths $(\mathrm{kHz})$.

Accordingly, error bounds are hard to generalize, since they depend on the specifics of the resonator. In a WR-340 waveguide resonator, disabling calibration or substituting the VNA and cabling causes relative differences in $Q_{0}$ close to $1 \%$ in most cases $(\max 2.7 \%)$ and close to $1 /\left(10 Q_{L}\right)$ for $f_{0}$.

The numerical perturbation analysis provides a qualitative confirmation of the conclusions above. It shows that, below a noise threshold, the uncertainty remains constant and independent on VNA noise. This residual uncertainty increases when transmission lines are included between the calibration plane and the resonator.

The results of the numerical perturbation analysis in our algorithm compare favorably with comparable numerical studies in other published works.

Finally, there is no indication of significant uncertainty from numerical matrix computations. The absolute relative differences in results obtained with the Moore-Penrose inverse and those obtained with normal equations are much smaller than those generated by other sources of uncertainty.

\section{REFERENCES}

[1] W. E. Courtney, "Analysis and evaluation of a method of measuring the complex permittivity and permeability microwave insulators," IEEE Trans. Microwave Theory Tech., vol. 18, no. 8, pp. 476-485, 1970.

[2] J. Breeze, Temperature and Frequency Dependence of Complex Permittivity in Metal Oxide Dielectrics: Theory, Modelling and Measurement, Springer Theses, Switzerland: Springer International Publishing AG, 2016.

[3] J. Baker-Jarvis, R. G. Geyer, J. H. Grosvenor, M. D. Janezic, C. A. Jones, B. Riddle, C. M. Weil, and J. Krupka, "Dielectric characterization of low-loss materials a comparison of techniques," IEEE Trans. Dielectr. Electr. Insul., vol. 5, no. 4, pp. 571-577, 1998.

[4] Z.-Y. Shen, C. Wilker, P. Pang, and W. L. Holstein, "High T/sub c/ superconductor-sapphire microwave resonator with extremely high Qvalues up to 90 K," 1992 IEEE MTT-S Microwave Symposium Digest, Albuquerque, NM, USA, 1992, pp.193-196, vol.1.

[5] R. J. Cameron, C. M. Kudsia, and R. R. Mansour, Microwave Filters for Communication Systems, 2nd ed., Microwave Theory \& Techniques, Hoboken, USA: John Wiley \& Sons Inc, 2018.

[6] I. E. Campisi and Z. D. Farkas, "High-gradient, pulsed operation of superconducting niobium cavities," SLAC, Rep. SLAC-AP-16, Feb. 1984.

[7] Asztalos, S.J. et al., "A SQUID-based microwave cavity search for darkmatter axions," Phys.Rev.Lett. 104, Jan. 2010.

[8] www.arpe.upc.edu, 2020.

[9] Python Software Foundation. Python Language Reference, version 3.8, Mar. 2019, Available: https://www.python.org.

[10] D. Kajfez, S. Chebolu, M. R. Abdul-Gaffoor, and A. A. Kishk, "Uncertainty analysis of the transmission-type measurement of Qfactor," IEEE Trans. Microwave Theory Tech., vol. 47, no. 3, pp. 367371, Mar. 1999.

[11] D. Kajfez, "Linear fractional curve fitting for measurement of high Q factors," IEEE Trans. Microwave Theory Tech., vol. 42, no. 7, pp. 11491153, Jul. 1994.

[12] D. Kajfez, Q Factor Measurements Using MATLAB, Massachusetts, USA: Artech House, 2011.

[13] N. Pompeo, K. Torokhtii, and E. Silva, "Dielectric resonators for the measurements of the surface impedance of superconducting films," Measurement Science Review, vol. 14, no. 3, pp. 164-170, Jun. 2014.

[14] K. Leong, and J. Mazierska, "Precise measurements of the Q factor of dielectric resonators in the transmission mode - Accounting for noise, crosstalk, delay of uncalibrated lines, coupling loss, and coupling reactance," IEEE Trans. Microwave Theory Tech., vol. 50, no. 9, pp. 2115-2127, Sept. 2002.

[15] D. Kajfez, "Numerical Determination of Two-Port Parameters from Measured Unrestricted Data," IEEE Trans. Instrum. Meas., vol. IM-24, no. 1, pp. 4-11, 1975.

[16] P. J. Petersan and S. M. Anlage, "Measurement of resonant frequency and quality factor of microwave resonators: Comparison of methods," $J$. Appl. Phys. , vol. 84, no. 6, pp. 3392-3402, Sep. 1998.

[17] A. Alimenti, K. Torokhtii, E. Silva, and N. Pompeo, "Challenging microwave resonant measurement techniques for conducting material characterization," Meas. Sci. Technol., vol. 30, p. 065601, 2019.

[18] K. Torokhtii, A. Alimenti, N. Pompeo, and E. Silva, "Uncertainty in uncalibrated microwave resonant measurements," in 24th IMEKO TC4 International Symposium, 2019, pp. 1-5.

[19] G. Steward, Introduction to Matrix Computations, Orlando, Florida, USA: Academic Press Inc., 1973.

[20] J. M. O’Callaghan, C. Sans, C. Collado, E. Canet, R. Pous, and J. Fontcuberta, "Accuracy considerations in microstrip surface impedance 
measurements," IEEE Trans. Appl. Supercond., vol. 7, no. 2, pp. 18691872, Jun. 1997.

[21] W. Altar, "Q Circles-A Means of Analysis of Resonant Microwave Systems," in Proceedings of the IRE, vol. 35, no. 4, pp. 355-361, Apr. 1947.

[22] Keysight Technologies, PathWave Advanced Design System (ADS), 2020.

[23] P. Krkotić, A. Aguasca, and J. M. O'Callaghan, "Small Footprint Evaluation of Metal Coatings for Additive Manufacturing," in 48th European Microwave Conference (EuMC), Madrid, Spain, Sep. 2018.

[24] W. B. Davenport Jr. and W. L. Root, An Introduction to the Theory of Random Signals and Noise, Hoboken, USA: John Wiley \& Sons Inc, Oct. 1987.

[25] B. Carlson and P. Crilly, Communication Systems: An Introduction to Signals and Noise in Electrical Communication, 4th ed., New York, NY, USA: McGraw-Hill, 2001.

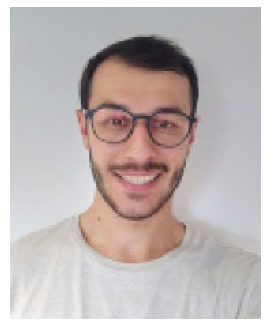

Patrick Krkotić (Student Member IEEE) was born in Dieburg, Hessen, Germany in 1992. He received the B.Sc. degree in physics and the M.Sc. degree in nuclear physics and nuclear astrophysics from Technische Universität Darmstadt, Hessen, Germany in 2014 and 2017 respectively. In 2015, he spend a year abroad studying astrophysics at the University of Bristol, England, United Kingdom. At present, he is pursuing a Ph.D. degree in physics at the Universitat Politècnica de Catalunya, Barcelona, Catalonia, Spain in collaboration with the ALBA Synchrotron Light Source, Barcelona.

Since 2016, he is involved in the Future Circular Collider design study for a new hadron-hadron collider at the CERN facility. His main work focuses on the usage of hightemperature superconductor coated conductors (HTS-CC) as an alternative coating for beam screens in future particle accelerators. His research interests include the development of radio-frequency based resonance-measurement systems for material characterisation, particularly surface impedance under the influence of high magnetic fields and synchrotron radiation. ORCID 0000-0003-1892-0350

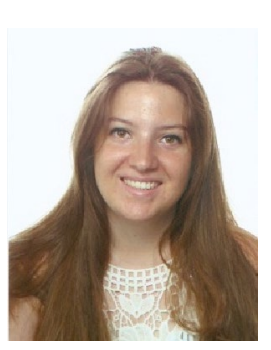

Queralt Gallardo was born in Barcelona, Catalonia in 1997. She received the B.Sc. degree in Telecommunications Technologies and Services Engineering from Universitat Politècnica de Catalunya (UPC), Barcelona, Catalonia, Spain in 2020. Currently, she is working for her Master's degree in Telecommunications Engineering at the UPC Barcelona.

In 2019, she spent six months as an intern at the ALBA Synchrotron Light Source, Barcelona where she successfully finished her thesis setting the milestones for this project.

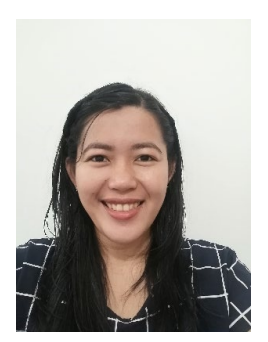

Nikki D. Tagdulang was born in Tangub City, Philippines in 1996. She received the B.Sc. and M.Sc. degree in physics from Mindanao State University-Iligan Institute of Technology, Iligan City, Philippines in 2016 and 2018 respectively. She is currently pursuing her Ph.D. degree at the Universitat Politècnica de Catalunya (UPC), Barcelona, Catalonia, Spain with the affiliation of ALBA Synchrotron Light Source, Barcelona.

Her main interests include radio frequency characterization of high-temperature superconductor coated conductors (HTS$\mathrm{CC}$ ) as alternative coatings to be used in the beam screen of the future circular collider. ORCID 0000-0002-6248-0906

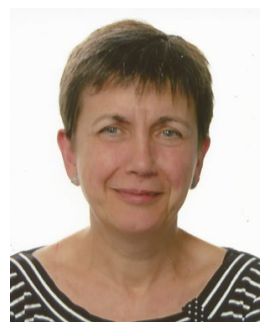

Montse Pont received the Ph.D. degree in physics from the Universitat Autònoma de Barcelona, Catalonia, Spain in 1990 in the field of materials science.

In 1994 she joined the recently created Synchrotron Light Laboratory in Barcelona and since then she has been working in accelerators for synchrotron light sources. She was at ANKA, (Karlsruhe, Germany) from 1998 to 2004. Thereafter, she is in the Accelerator Division of the ALBA Synchrotron Light Source in Barcelona. Her field of expertise includes magnet design and construction, and commissioning and operation of synchrotron light sources. ORCID 0000-0003-4830-2692

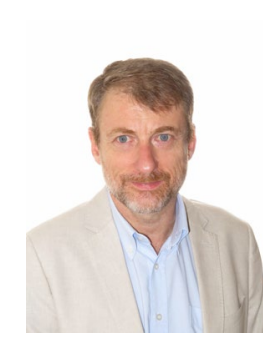

Juan M. O'Callaghan (Senior Member IEEE) obtained the telecommunication engineering degree from Universitat Politècnica de Catalunya (UPC), Barcelona, Catalonia, Spain in 1987 and the Ph.D. degree from the University of Wisconsin, Madison in 1993.

In 1989 he worked at Honeywell's Systems Research Center (Bloomington, Minnessota) working on noise measurement methods for FETs a Ka band. In 1993 he became a tenure-track faculty and became Full Professor in 2003 at UPC where he is currently working. He is an expert in microwave devices and systems. He is Principal Investigator (PI) on several projects with public and private funding. He has published 66 articles in international journals and holds 6 patents (ORCID 0000-0002-2740-0202, see full record at https://futur.upc.edu/JuanManuelOcallaghanCastella). $\mathrm{He}$ is co-founder and technological adviser of Miwendo Solutions (https://www.miwendo.com/ ). His research interests include microwave devices, materials and microwave biomedical applications. 